\title{
A Knowledge-Based Space Planning System
}

Article in Architectural Science Review · March 1992

DOI: $10.1080 / 00038628.1992 .9697305$

CITATIONS

6

3 authors, including:

Halime Demirkan

Bilkent University

42 PUBLICATIONS 837 CITATIONS

SEE PROFILE
READS

51

Mustafa Pultar

Bilkent University

7 PUBLICATIONS 82 CITATIONS

SEE PROFILE 


\section{Contents, March 1992}

Ediwrial: Solving Yesterday's Problems

A Knowledge-Based Space Planning System

H. Demirikan, M. Pultar, and B. Özgü̧̧

Keyless Data Acquisition in Construction

F.-S. Liou

Road Traffic Noise Intrusion - Retrofitting and

Compensation - An Architectural Future

P.-A. Johnson

Design Problems and Evaluation Procedures

Explorations of Technicological Issues in the

Design Sudio Using CAAD

Letters to the Editor

S. Coldicuth. T. Heach and K. A. Rose
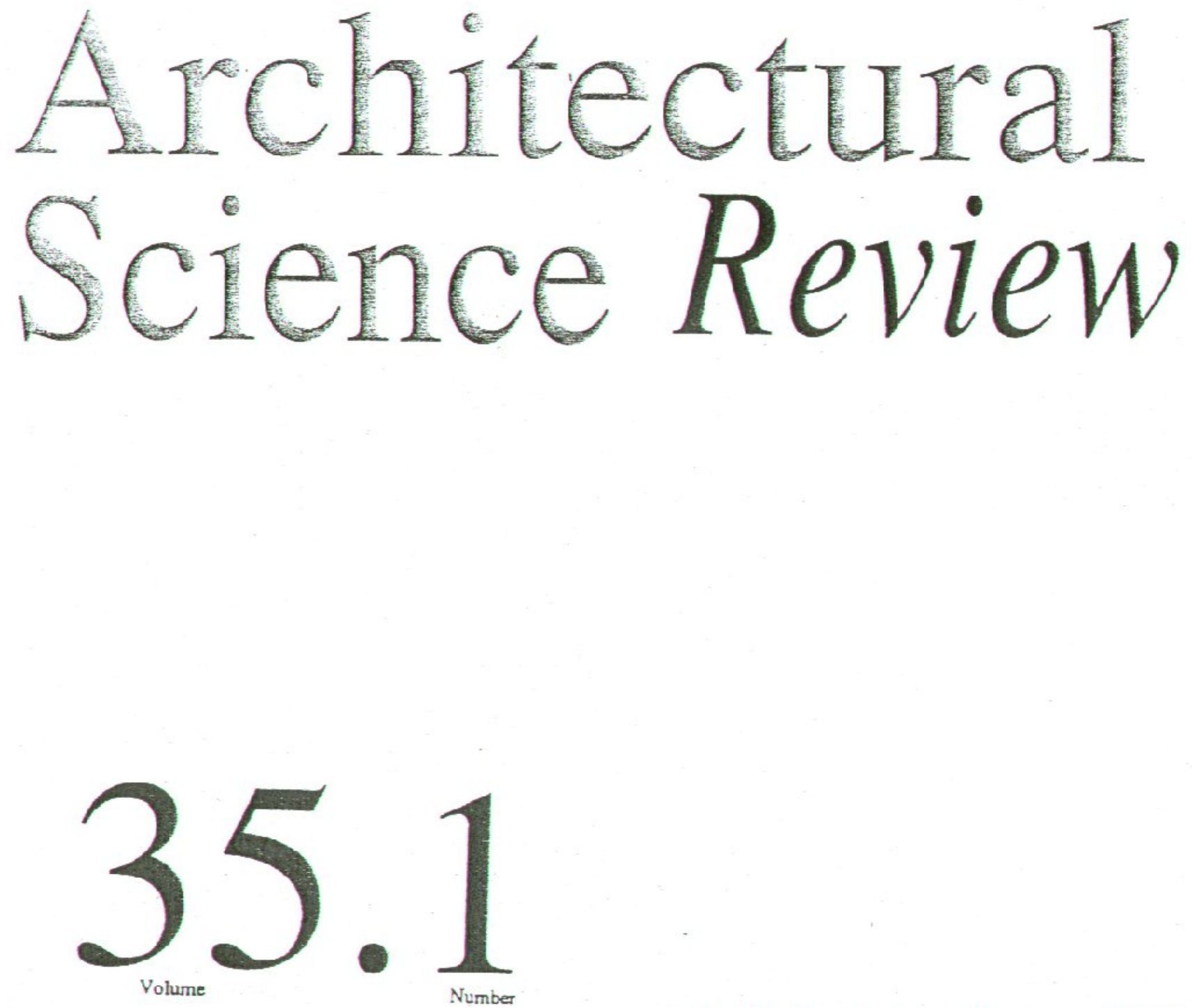


\title{
A Knowledge-Based Space Planning System
}

\section{Halime Demirkan, Mustafa Pultar and Bülent Özgüç}

\author{
Faculty of Art, Design and Architecture, Bilkent University, Ankara, Turkey
}

Knowledge-based systems are potentially powerful tools for improving the results of automated or interactive design procedures. Expert Space Planner is a prototype of such a system that uses production rules in the spatial planning of panel houses.

\section{Introduction}

Due to their nature, architectural design problems are yet to get their share from the technological developments in computer science. Knowledge-based systems promise to be powerful tools in this field (Gero,1985). If low-level design decisions can be made by the computer, within a given time slice, there should be an improvement in the quality of a design in an interactive environment since more time can now be allocated for more complex decisions.

Space planning can be considered as a subtask of the design process in general. It is concerned with the satisfactory arrangement of objects in space in order to meet a set of objectives. In architectural design the objects are the spaces themselves. Space planning may involve the determination of the dimensions of objects and of the spaces between them. It may also include the organization of objects and spaces to meet objectives.

There are a variety of possible ways of encoding knowledge. These include state-space representations, logic-based representations, semantic nets, production systems and frame systems. Design has the characteristics of being knowledge rich. Expert Space Planner (ESP) is a prototype of a knowledgebased system that has a set of production rules using reasoning proccdures. Control is achieved by means of production rules. In the implementation of the space planning system, backtracking is accomplished by the interactive intervention of the human operator.

It should be noted that, implementing ESP would not exclude the role of the human designer. ESP would simply assist the human designer, by relieving him from performing the more trivial tasks.

\section{Conceptual Framework of ESP}

The general structure of ESP is schematized in Figure 1. There are two major components of the system: the planning and the database components. Each of these components is made of subcomponents: the planning component consists of design goals, production rules and a planning model as shown in Figure 1. The database provides the basis for the decisions made by the planning components and links the objects in specified ways. The user interface facilitates the communication between the real world and the computer.

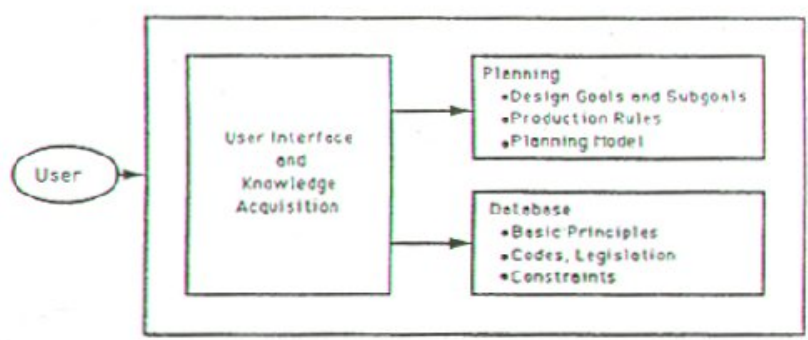

Figure 1. Conceptual framework of Expert Space Planner $(E S P)$.

This study is aimed at the space planning of industrialized construction of houses produced by the Small Panel System . The system panels are $120 \mathrm{~cm}$. in width and 240,260 or 280 $\mathrm{cm}$. in height. The thicknesses of the panels are $6 \mathrm{~cm}$. for interior walls and $8 \mathrm{~cm}$. for exterior walls. 


\subsection{Design Goals and Subgoals}

Goals are simply atuributes that the designer wants to see exhibited in the final state of the design. A goal may be represented as certain qualities or parts that are to be present in the final artifact, such as a dining room, a sleeping room, etc. in a house. Alternatively, a goal may be expressed as a requirement that there must be particular relationships between elements, such as a dining room is to be affiliated with the kitchen. Furthermore, a goal may be a requirement possessed by an element such as the shape of a room is to be rectangular. Goals may be expressed as high-level requirements that need translating into system goals, as in the requirements of building codes and regulations (Coyne, 1985).

Goals are almost never mutually exclusive. In trying to reach a successful solution, it may be discovered that the condition required to reach one solution is contradictory to another goal. It is then necessary to find a means of satisfying the first goal that does not produce a conflict with the second one (Coyne, 1985).

In ESP, certain goals have been set as described below:

Shape of the spaces: The shape of the spaces should be rectangular or L-shaped. Two adjacent walls should be perpendicular to each other. This is a goal that needs to be achieved because of the construction system. Spaces should not have curved walls as this shape is not possible in the small panel system.

Minimum space requirements: Another goal is to satisfy the minimum area requirements of the spaces as set by building codes (Building Regulation). The minimum space requirements are:

$\begin{array}{ll}\text { Living room: } & (2.80 \times 4.00) \mathrm{m} . \\ \text { Bedroom: } & (2.10 \times 2.80) \mathrm{m} . \\ \text { Kitchen: } & (1.50 \times 2.00) \mathrm{m} . \\ \text { Bathroom: } & (1.20 \times 2.00) \mathrm{m} \\ \text { WC: } & (0.90 \times 1.20) \mathrm{m} . \\ \text { Corridor: } & (1.00 \times 1.20) \mathrm{m} .\end{array}$

Minimum number of spaces: There should be at least a kitchen, a bathroom, a WC and a living room in a house. This is the minimum number and composition of spaces that should be presented within a living unit.

Requirement of an exterior wall: Spaces should contain at least one exterior wall except for corridors. In order to use daylight, each space should have a window. A corridor is a space in which circulation occurs, it should be connecting various other spaces. Therefore, an exterior wall recommendation is not necessary.

Subgoals are not sequential and all subgoals must not necessarily be satisfied in order to achieve a given goal (Kalay, 1985). The subgoals of the ESP have been set as follows:

Type of equipment in the wet cores: In the spatial layout, there should be certain equipment allocated within various service cores. These equipment consist of a sink in a kitchen, a bathtub or a sitbath, a basin and a WC in a bathroom and a WC and a small basin in an additional WC. These are the minimum number and types of equipment that should be presented within a certain wet core. This is a subgoal of the minimum area requirement of the spaces.

Types of furniture: The furniture located in spaces constitute, in fact, the subgoals. They should be located properly within a given space. A conflict may arise again, if the space does not allow the provision of an appropriate number of furniture. This is a subgoal of the shape of the spaces.

\subsection{Production Rules}

Each transformational rule has a precondition that relates to the global database and describes when the rule can be used. A rule is a condition/action of the form:

\section{if $\mathbf{A}$ then $\mathbf{B}$}

where $\mathrm{A}$ is some condition that has to be satisfied by the data in the database before the rule can be applied, B is the action to be taken when the condition $\mathrm{A}$ is satisfied.

The rules do not need to have a meaning. Each rule could be identified arbitrarily. Example of such an action in the design of a building could be the placement of the dining room next to the kitchen.

The space layout problem has been partitioned into independent subproblems. The rules can be considered as ordered subsystems. Each subsystem takes as input the output of the previous system. The ordering of the rules provides an appropriate sequence of design.

When new knowledge is provided, the relationship of the existing rules must be checked. The rule order is dependent on the knowledge of the designer.

Rules are organized into groups such that those which are of the same hierarchical level are put into the same group. The grouping can take the form of a schedule.

There are three types of rules that are used in ESP. In the first type, each rule may be used as many times as necessary during the design process. Every time the user interactively selects the Space Menu in order to draw a space, the dimensions of the space should be specified. In the second type, any rule can be checked for true design decisions. After the dimensions are specified, they are checked whether the area of the space satisfies the minimum area requirements, as set previously. In the third type, if all of the rules have been applied from the previous set, the system starts to apply the rules from the following group (Coyne, 1985). After the space planning is completed, either the equipment or the furniture should be selected. The objects are checked to see whether they can be properly placed within the designed spaces.

\section{Database}

The database is the second component of a knowledge-based system. It maintains the integrity and provides the basis for the decisions made by the planning component. A database is a collection of regularly formatted data that is accessed by more than one person and/or is used for more than one person (Frost, 1986). The part of a knowledge base that can be represented in a uniform format can be stored in a database.

Every object has both descriptive and functional knowledge associated with it (Kalay, 1985). The descriptive knowledge is made up of properties of the object such as form, geometrical location. The database of ESP expands as the user runs it. New data files are created interactively as design progresses. The type of space and the dimensions related to it are stored in a file. 
The functional knowledge describes how the object should be treated with regard to its various parameters. These relationships are classified by Kalay (1985) as:

part-whole relationships,

master-instance relationships,

group relationships.

Part-whole relationships link objects in a rank. A door, for example, is a part of a wall, which in turn is a part of a house. If a door is going to be erased, since it is a part of a panel, it should be replaced with a simple panel in order to enclose the space.

Master-instance relationships state that every object belongs to a class of objects defining its own set. In ESP there is a master-instance relationship between a window and a panel. When user selects a window to be drawn from the menu, it is within a panel.

Group relationship is a kind of link in which any change affects all the members of the group. For example, if a wall is rotated. the connecting walls must also be rotated with the same angle.

\subsection{Codes and Constraints}

In addition to objects and their attributes, the database also contains basic principles, provisions of codes, legislation and other similar constraints. These should be continuously cvaluated by the software.

Some examples of such constraints used in ESP are the following: According to the legislation minimum space dimensions for different parts of a house are stated. If the user inputs values less than these, the program generates messages and requests for new values. Other codes may be used for different purposes.

One side of the house may not be more than six panels, or $7.20 \mathrm{~m}$. The reason for this is that the roof used in this construction system cannot be manufactured to cover a bigger span. Various other constraints are also built into the system.

\section{General Structure of ESP}

In order to use the knowledge-based space planing system successfully, a set of rules are to be followed interactively. The main menu is the 'Space Planning Menu' that has two basic components; the project elements and the furnishing elements. The project elements consist of the spaces and the basic building elements. The furnishing elements consist of equipment for the wet cores and the furnitures that could be used in a house.

The graphic screen is divided into three windows. The space planning is achieved on a grid drawn in the first window. The second window is reserved for menus that may appear according to the current state of the execution. Third window is for interactive data input.

During the initial phase of execution a grid is drawn on the first window that has alphabetic characters on the horizontal, and numeric characters on the vertical axes. In the second window, a 'Space Planning Menu' appears. The options on this menu are: (1) Project Elements; and (2) Furnishing Elements. The user specifies his options in the third window (Figure 2).
If project elements option is selected, 'Project Elements Menu' appears in the second window. It has 'Spaces' and 'Building Elements' as its options.

If furnishing elements option is selected 'Furnishing Elements Menu' appears. This menu has 'Equipments' and 'Furnitures' as its options.

An error recovery program has been developed for maintaining the flow of the program in the case of erroneous data. Data of wrong format or magnitude, when possible, is automatically corrected.

\subsection{Spaces Subsystem}

This function of the system lists the available spaces in a house and requests a choice. The user provides the code of the space, dimensions based upon the number of panels and the reference point of the space. The designer then may translate it to another location, fix or erase it according to his evaluations.

\subsection{Building Elements Subsystem}

A menu that consists of 'Stairs', 'Wardrobes', 'Chimneys', and 'Panels' appear under the 'Building Elements' option. Even though expandable, the current state of the system would give the following options when one of the menu items is selected.

Siairs: Four types of precast staircases are displayed. The user should select a type, and specify its location and coordinates.

Wardrobes: Seven types of prefabricated wardrobes are displayed. The user should specify the type, coordinates and the comer which should intersect with the grid.

Chimneys: Four types of chimneys are displayed. The user should specify the type, coordinates and reference point interactively.

Panels: Simple panel, window panel, outdoor and indoor panels are displayed. The user should specify the panel type and its location. The user may erase items previously drawn through the same procedure.

\subsection{Equipments Subsystem}

This part consists of procedures for the shower tub, basin, sitbath tub, water closet, sink, squat water closet, small basin, etc. The user may draw or erase the selected equipment. When the type of the equipment is specified, a new menu appears. This menu contains the drawings of the equipment according to their possible placements. An equipment is interactively selected and located.

\subsection{Furniture Subsystem}

This function consists of bed, double bed, easy chair, double easy chair, sofa, sideboard, low table, dining table, refrigerator, cooker, etc. The user may draw or erase a specified unit. The selection and placement is done similar to the equipment subsystem. 


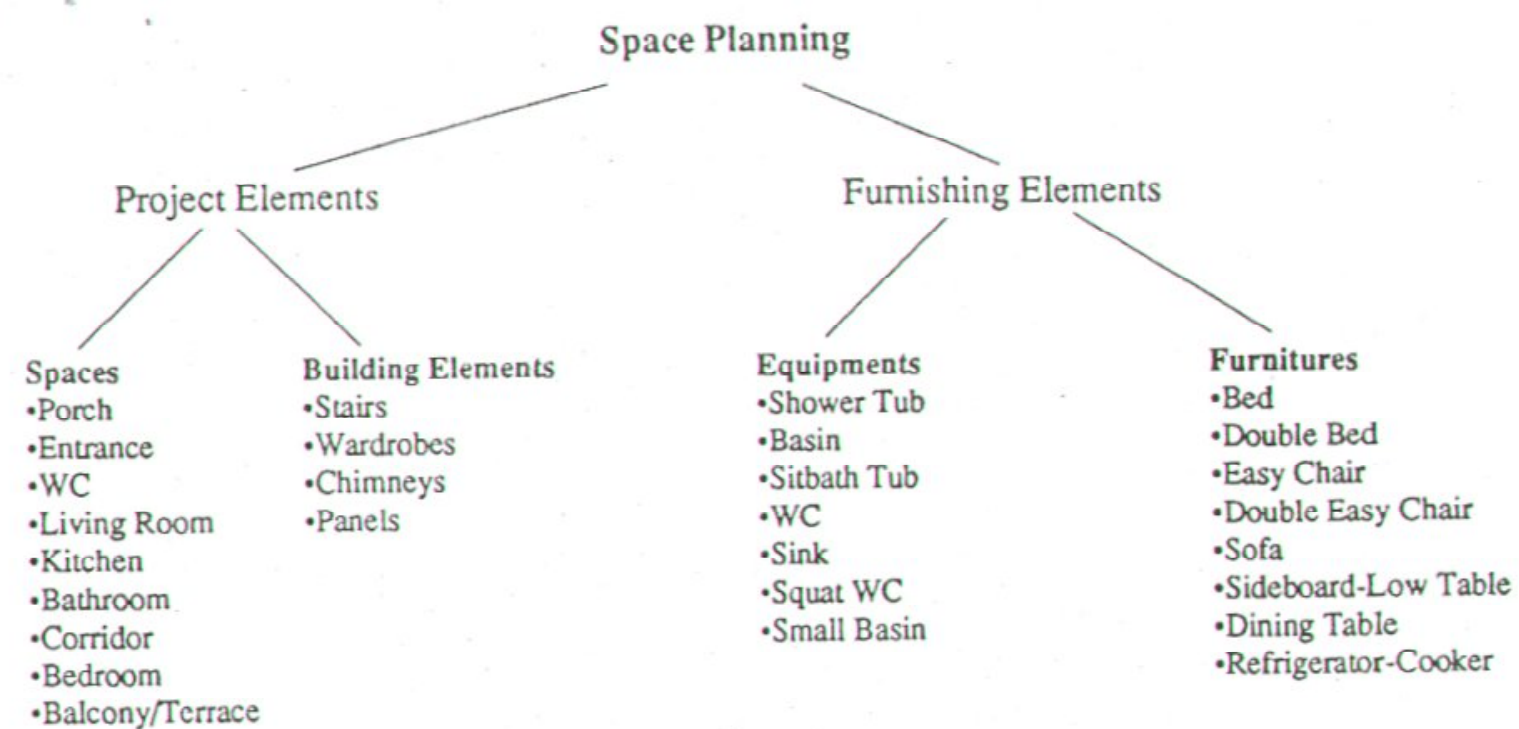

Figure 2. Scheme of the system.

An interactive session with ESP is carried out at various levels. At the first level, spaces are selected and are drawn on the grid. In order to integrate the spaces, some may be translated while new ones are added. Previously described goals and subgoals are tested as the design progresses. Each goal should be satisfied before the current level is completed. The system will also warn the user if there is a conflict with the legislation or the construction system.

Forward and backward tracking is possible throughout ESP. The designer may select the options from building or furnish- ing elements subsystems at the next or previous level. It is also possible to shift from one level to another during the design process. The database expands as the process advances. Furnitures and equipments are located according to their appropriate spaces, locational attributes and compatibility.

In Figure 3, a $110 \mathrm{~m}^{2}$ house with three bedrooms is shown. In Figure 4, a bungalow type house with an area of approximately $70 \mathrm{~m}^{2}$ is shown.

Both plans have been developed exclusively by the system in order to demonstrate its capabilities.

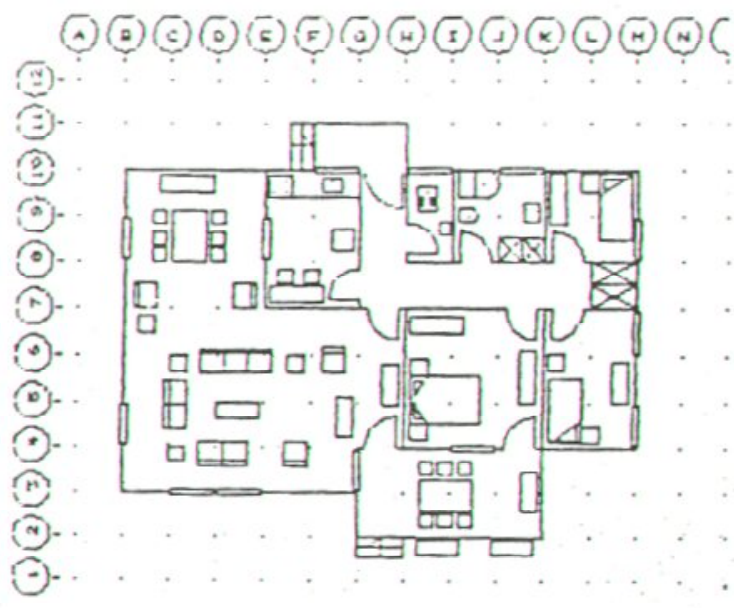

Figure 3. Three-bedroom plan type

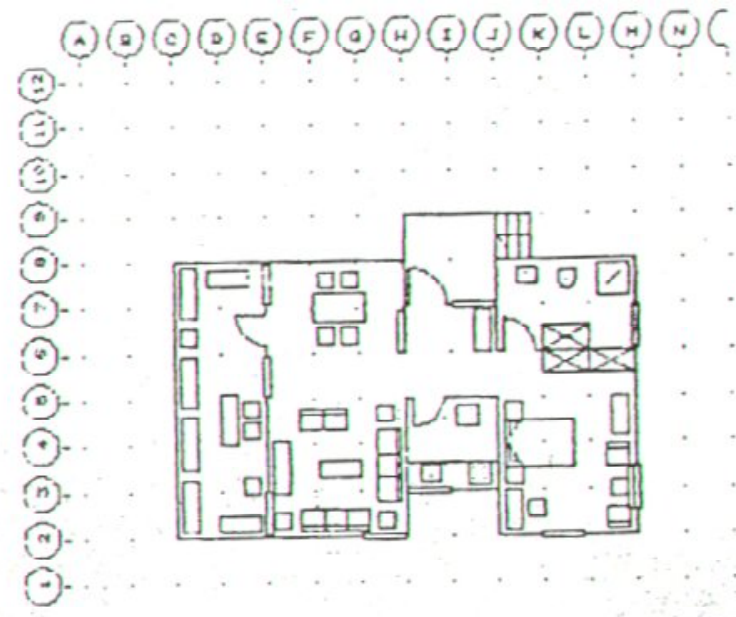

Figure 4. Bungalow plan type. 


\section{Conclusions}

ESP develops altemative space planning models that can be considered as the initial phase of design problems. The time saved in the planning phase can be used to develop alternative models. The space planning alternative that can be visually presented on the screen helps to instantly correct the erroneous designs. The knowledge which is accumulated with the experience of the designer can be coded and retrieved. Each designer may code his own experience in order to develop new expert systems. This is achieved by modifying, deleting or adding new goals and constraints through the individual experiences.

The plan that is designed could be used to inform the designer/client (user) and builder about the house. It could also be used to give a global idea about the cost of the building. Furthermore, it would give a chance to the client (user), builder and designer for making changes on the design without violating the goals.

The same plans and database can be used by the electrical, mechanical and civil engineers simultaneously. They can generate their own designs without loosing time. The teamwork can be fruitful by exchanging ideas in the preliminary design phase. ESP tries to show that there is a uniformity between design operations.

\section{References}

1. Coyne, R.D., Knowledge-based Planning Systems and Design: A Review, Architectural ScienceReview, Vol. 28, No. 4 (1985) pp. 95-103.

2. Frost, R.,Introduction to Knowledge Based Systems, New York: Macmillan Publishing Company, 1986.

3. Gero, J.S., Editorial - Special Issue on Exper Systems in CAD, Computer-Aided Design, Vol. 17, No. 9 (1985), pp. 396-398.

4. Kalay, Y., Redefining the Role of Computers in Architecture: from Drafting/Modeling Tools to Knowledge-Based Design Assistants, Computer-Aided Design, Vol. 17, No. 7 (1985), pp. 319-328. 